\title{
MONITORAMENTO DE COLEOBROCAS ASSOCIADAS À MANGUEIRA NO MUNICÍPIO DE JOSÉ DE FREITAS, ESTADO DO PIAUÍ ${ }^{1}$
}

\author{
JEAN KELSON DA SILVAPAZ², PAULO ROBERTO RAMALHO SILVA, LUIZ EVALDO DE MOURAPÁDUA ${ }^{4}$, \\ SERGIO IDE $^{5}$, EULÁLIA MARIA SOUSA CARVALHO ${ }^{6}$, SÁVIO SILVEIRA FEITOSA7 $^{7}$
}

RESUMO - Este trabalho foi desenvolvido de junho de 2004 a maio de 2005, objetivando o reconhecimento de espécies de coleobrocas (Insecta: Coleoptera) que se encontram associadas a pomar comercial de manga (Mangifera indica) das variedades Tommy Atkins, Keitt, Kent e Palmer, no município de José de Freitas, Estado do Piauí. Foram instaladas duas armadilhas etanólicas modelo Carvalho $47 \mathrm{em}$ cada área das referidas variedades e realizadas coletas semanais. As coleobrocas foram separadas dos resíduos vegetais, secas sob lâmpada e quantificadas. Posteriormente, exemplares de cada morfoespécie coletada foram identificados. Coleobrocas das famílias Bostrichidae, Cerambycidae, Curculionidae estão associadas às variedades de manga cultivadas no município de José de Freitas, Estado do Piauí, onde se destacam as espécies Hylettus seniculus (Germar) (Coleoptera: Cerambycidae), Hypothenemus sp. 1 e sp. 2. (Coleoptera: Curculionidae: Scolytinae), apresentando maior expressão numérica e variedade de morfoespécies durante o período de menor precipitação pluviométrica, fator correlacionado positivamente à família Cerambycidae.

Termos para Indexação: Bostrichidae, Cerambycidae, Curculionidae, Mangifera indica.

\section{MONITORING OF COLEOBORERS ASSOCIATED TO MANGO TREE IN JOSÉ DE FREITAS CITY, STATE OF PIAUÍ, BRAZIL}

\begin{abstract}
This research was developed from June 2004 to May 2005, under field conditions, aiming the recognition of species of coleoborers that are associated to commercial mango orchard (Mangifera indica L.) of varieties Tommy Atkins, Keitt, Kent and Palmer, in José de Freitas city, state of Piauí. It was installed two ethanolics traps model Carvalho 47 in each area of the related varieties and weekly collections were realized. Coleoborers were separated of the vegetal residues, dried under light bulb and quantified. Samples of each morphspecies were collected and mounted in entomologic pins then sent for identification. Coleoborers of the families, Bostrichidae, Cerambycidae and Curculionidae are associated to the varieties of mangoes cultivated in José de Freitas city, state of Piauí, highlighting the species Hylettus seniculus (Germar) (Coleoptera: Cerambycidae), Hypothenemus sp. 1 and sp. 2 (Coleoptera: Curculionidae: Scolytinae), presenting bigger numerical expression and variety of morphspecies during the period of lower pluviometric precipitation, this factor presenting positive correlation with the Cerambycidae family.
\end{abstract}

Index Terms: Bostrichidae, Cerambycidae, Curculionidae, Mangifera indica.

\section{INTRODUÇÃO}

Nos trópicos, os coleópteros são dominantes e responsáveis por grandes prejuízos em espécies vegetais, sobretudo espécies das famílias Curculionidae e Cerambycidae, pois estes desempenham papel importante na degradação da madeira (Gray, 1972). Esses insetos são comuns nas regiões tropicais e, embora sejam considerados de importância secundária, só atacam árvores vivas que apresentem alterações nas suas condições fisiológicas, mas podem causar grandes prejuízos, sobretudo pelas aberturas de galerias e manchamento da madeira (Hosking, 1977). Nos curculionídeos, a base da alimentação é deficiente em vitaminas essenciais do grupo B e esteróis, cuja ausência é compensada por uma dieta rica em nitrogênio fornecida por fungos simbiontes que as sintetizam partindo de nutrientes absorvidos das galerias (Batra, 1963).

No Brasil, acredita-se que coleobrocas venham a causar problemas, pois desde levantamentos que tiveram início na década de 70 e que permanecem até os dias atuais, estes aumentaram tanto em número de espécies como de indivíduos (Flechtmann et al., 1999). A presença de espécies de coleobrocas está-se tornando relativamente comum em plantações florestais, o que ainda não se aplica aos pomares frutíferos, porém sua importância e abundância têm aumentado, principalmente para as espécies de besouros da ambrósia, o que pode vir a tornar-se um grande problema para a fruticultura (Flechtmann et al., 2001). Muitos trabalhos, como os de Zanuncio et al. (2005), Flechtmann \&

'(Trabalho 103-07). Recebido em: 17-04-2007. Aceito para publicação em: 07-03-2008. Parte da Dissertação do primeiro autor apresentada ao Programa de Pós-graduação em Agronomia- PPGA - CCA/UFPI, como requisito para a obtenção do título de Mestre em Agronomia.

${ }^{2}$ Professor,Mestre do Departamento de Fitotecnia,CCA/UFPI, Campus Socopo, CEP 64049-550, pazjks@yahoo.com.br.

${ }^{3}$ Professor Doutor do Departamento de Fitotecnia do Centro de Ciências Agrárias da Universidade Federal do Piauí, Campus Socopo, CEP 64049-550, pauloramalho@ufpi.br.

${ }^{4}$ Professor Doutor do Departamento de Fitotecnia do Centro de Ciências Agrárias da Universidade Federal do Piauí, Campus Socopo, CEP 64049-550, lempadua@ufpi.com.br.

${ }_{5}^{5}$ Pesquisador Doutor do Instituto Biológico de São Paulo. ide@biologico.sp.gov.br.

${ }^{6}$ Professor Adjunto Mestre do Departamento de Fitotecnia do Centro de Ciências Agrárias da Universidade Federal do Piauí, Campus Socopo, CEP 64049550, eulaliac@bol.com.br.

${ }^{7}$ Engenheiro Agrônomo, Mestre, Instituto Nacional de Colonização e Reforma Agrária (Incra/Sede/DF).savio.feitosa@incra.gov.br. 
Gaspareto (1997) e Abreu et al. (2002), demonstram a importância de coleobrocas no Brasil, danificando tanto árvores de pé, quanto madeira estocada em serrarias. Isso é preocupante, pois, segundo Vité (1971), o controle químico de coleópteros da subfamília Scolytinae é um evento "post-mortem", pois o tratamento é feito tarde demais para as árvores infestadas, uma vez que a aplicação localizada nunca atinge a população em trânsito. Além disso, o controle direto não leva em conta o controle natural (parasitóides, predadores e competidores) e há indícios de que o controle químico neutraliza as competições intra e interespecíficas.

A maneira mais eficiente de manejo é o monitoramento sistemático, para que possam ser tomadas medidas de controle quando necessárias. Silveira Neto et al. (1976) afirmaram que o levantamento de insetos é de fundamental importância em estudos ecológicos, pois é praticamente impossível contar todos os insetos de um habitat, e estes estudos só poderão ser realizados mediante estimativas de população por meio de amostras. A influência ou a precisão de uma amostra é o resultado do produto dos componentes pessoais, estatísticos, mecânicos e econômicos. Não existe um método de amostragem universal, e freqüentemente um método empregado para um determinado inseto não se aplica a outro; às vezes, um método não se emprega ao mesmo inseto em condições diferentes. É preciso estabelecer para cada caso, mediante amostragem prévia, a melhor e a mais eficiente maneira de efetuar um levantamento populacional e, dessa forma, transformá-lo em uma ferramenta importante no combate às pragas.

No Brasil, muitos estudos com coleobrocas abrangem levantamentos populacionais que, por vezes, são correlacionados a dados climáticos e de vegetação, com ou sem a aplicação de índices faunísticos, utilizando-se de armadilhas de impacto, cujo atrativo mais comum é o álcool etílico (Carvalho et al., 1996; Dall' Oglio \& Peres Filho, 1997; Morales et al., 1996). Ferraz et al. (1999; 2000) utilizaram a armadilha etanólica modelo Carvalho 47 em experimentos realizados em pomar de Citrus e plantios de Eucalyptus spp. no Estado do Rio de Janeiro. Carvalho (1984) já havia realizado experimento semelhante em plantios de Eucalyptus spp. no mesmo Estado. Dall'Oglio \& Peres Filho (1997) monitoraram área de plantio de seringueira em Itiquira, Estado do Mato Grosso, com armadilha etanólica de ação semelhante. O uso do álcool etílico,como princípio atrativo para monitoramento e controle deste grupo de insetos, foi recomendado por Nakano \& Leite (2000), sendo que eles afirmam que plantas atacadas entram em fermentação alcoólica, em conseqüência da decomposição de matéria vegetal causada por fungos que penetram pelas galerias dos ramos atacados e, dessa forma, atraem outras coleobrocas. Outros métodos podem ser empregados para monitorar coleópteros, como o usado por Garcia \& Corseuil (1999), que estudaram espécies de coleópteros em cultivares de pessegueiros no município de Porto Alegre, Estado do Rio Grande do Sul, utilizando frasco caça-moscas contendo suco de frutas; todavia, as armadilhas etanólicas têm demonstrado maior eficiência para monitorar este grupo de insetos.

O monitoramento de pragas em áreas produtoras é de fundamental importância para que se possa ter informações seguras para a implantação de programas de manejo integrado de pragas e diminuição dos custos de produção.

Este trabalho teve como objetivo monitorar área produtora de manga (Mangifera indica), localizada no município de José de Freitas, Estado do Piauí, e desta forma obter informações sobre a dinâmica populacional e registrar a ocorrência de espécies de coleobrocas associadas a esta cultura.

\section{MATERIAL E MÉTODOS}

O experimento foi conduzido de junho de 2004 a maio de 2005, em pomar comercial de manga, em uma área de 100 hectares (123 plantas/ha), das cultivares Tommy Atkins, Keitt, Kent e Palmer no município de José de Freitas, Estado do Piauí. O município está na latitude: $04^{\circ} 50^{\prime} \mathrm{S}$ e longitude: $42^{\circ} 41^{\prime} \mathrm{W}$, inserido em clima tropical megatérmico, muito quente e subúmido do tipo seco (Lima, 2002). O pomar não foi submetido a tratos culturais, e as plantas, que possuíam em média oito anos, permaneceram, na sua maioria, no estado vegetativo durante todo o período do experimento. Foram instaladas em plantas da região central de cada cultivar componente do pomar duas armadilhas etanólicas modelo Carvalho 47, utilizada por Carvalho et al. (1997) e Ferraz et al. (2000), a 1,5 m de altura do solo e distanciadas $60 \mathrm{~m}$ uma da outra. Essas armadilhas continham álcool comercial ( $92,8^{\circ}$ INPM), que era renovado semanalmente por ocasião de cada uma das 54 coletas ao longo do período de monitoramento. Os frascos com as coleobrocas eram encaminhados a laboratório, onde as coleobrocas eram separadas dos resíduos vegetais, secas sob lâmpadas e identificadas em nível de gênero e/ou espécie. Os insetos foram quantificados, e exemplares de cada morfoespécie coletada foram montados em alfinetes entomológicos e identificados em nível de espécie. Foram construídos tabelas e gráficos para registrar a ocorrência das coleobrocas e a associação das espécies a cada cultivar estudada, bem como registrar a variação mensal durante o período de monitoramento. Foi realizada correlação linear simples (r) para analisar a influência da precipitação pluviométrica e a flutuação populacional das famílias de coleobrocas referentes ao período de monitoramento. A significância foi testada através do teste " $t$ " de Student, a nível de $10 \%$ de probabilidade. A identificação das espécies Bostrichidae e Curculionidae foi feita por Sergio Ide, do Instituto Biológico de São Paulo, e as espécies de Cerambycidae por Ubirajara R. Martins do Museu de Zoologia da USP.

\section{RESULTADOS E DISCUSSÃO}

Durante o período de monitoramento, foram realizadas 54 coletas e capturadas 3.317 coleobrocas nas áreas cultivadas com as quatro cultivares. As coleobrocas pertenciam às famílias Bostrichidae, Cerambycidae e Curculionidae. Foram capturados 2.988 indivíduos da família Curculionidae e registrada a ocorrência de 13 espécies distintas desta família. Cerambycidae foi o segundo maior grupamento, com 324 coleobrocas capturadas e distribuídas em 18 espécies distintas. Foram coletados ainda cinco indivíduos da família Bostrichidae, de espécie única (Tabela 1). 
Percentual e freqüência das famílias e das espécies

A Tabela 2 apresenta o percentual e a freqüência das famílias e das espécies durante o período de monitoramento. A família Curculionidae representou 90,1\% das coleobrocas capturadas, e Hypothenemus sp. 2 representou 52,8\% de Curculionidae e $47,5 \%$ das coleobrocas capturadas. Hypothenemus sp. 1 e Xyleborus sp. 4 representaram ainda 16,8 e $10,4 \%$, respectivamente, dos curculionidae capturados. Entre os curculionídeos, registrou-se a ocorrência de uma coleobroca pertencente ao mesmo gênero de Marshallius anacardii, descrita por Lima (1979) como praga nociva em anacardiáceas no Estado de Pernambuco, e Platypus parallelus (Fabr.), da subfamília Platypodinae. A supremacia de indivíduos da família Curculionidae neste trabalho pode ser atribuída ao fato de estes serem facilmente atraídos por armadilhas etanólicas, o que é relatado por Ferraz et al. (1999). Esses resultados também correspondem ao encontrado por Carvalho (1984), que coletou maior número de indivíduos das famílias Curculionidae e Cerambycidae em plantios de Eucalyptus urophylla (Myrtaceae) e Eucalyptus saligna (Myrtaceae) no município de Salto, Estado de São Paulo, com armadilhas luminosas modificadas, tendo também álcool etílico como atrativo. Em relação à família Cerambycidae, 9,8\% das coleobrocas capturadas pertenciam a esta família, destacando-se Hylettus seniculus (Germar) e Compsoibidion vanum (Thomson) com 47,2 e 13,6\%, respectivamente, das coletas desta família. Entre os Cerambycidae capturados, foi registrada ocorrência de Chlorida festiva (L.), citada por Gallo et al. (2002) como praga-chave na cultura da manga, sendo que esse inseto representou $4,3 \%$ dos cerambicídeos capturados. Bostrichidae representou $0,15 \%$ das coleobrocas capturadas e teve como seu único representante Bostrichopsis unicinata (Germar).

\section{coleobrocas \\ Ocorrência e flutuação populacional das famílias de \\ Espécies de Curculionidae estiveram presentes durante} todo o período de monitoramento, e seu pico populacional ocorreu durante o mês de maio de 2005, com 755 indivíduos capturados, e as menores ocorrências foram nos meses de novembro de 2004, com 76, e abril de 2005, com 90 (Figura 1). Espécies de Cerambycidae tiveram maior ocorrência no mês de fevereiro de 2005, com 83 indivíduos capturados, e apenas 8 em junho de 2004 (Figura 2). Bostrichidae não apresentou grandes variações durante o período de monitoramento e não foram registradas ocorrências de indivíduos dessa família durante os meses de junho, julho, agosto, setembro e dezembro de 2004, e no período de março a maio de 2005 (Figura 3).

Esses resultados assemelham-se aos encontrados por Dall'Oglio \& Peres Filho (1997), apesar de não registrarem a flutuação populacional, ao monitorarem área de plantio de seringueira em Itiquira, Estado do Mato Grosso, com armadilha etanólica. Os autores coletaram indivíduos das famílias Curculionidae e Cerambycidae. Afirmaram, ainda, que foram identificados curculionídeos dos gêneros Cnesinus, Corthylus, Cryptocarenus, Microcorthylus, Premnobius, Sampsonius, Spermophthorus, Theoborus, além dos gêneros Hypothenemus e Xyleborus, encontrados também neste trabalho. Esses autores citam ainda os cerambicídeos Achryson surinamum, C. festiva, Trachyderes succintus succintus, Eburodacrys sp, encontrados também nesta pesquisa, além de Estola sp., Hesychotypa subfasciata, Neoclytus pusillus e Oreodera acrumnosa. Os mesmos autores registraram ainda o bostrichídeo Bostrichopsis unicinata, também encontrado nesta pesquisa.

Com presença de coleobrocas durante todo o período de monitoramento, o pico populacional dos indivíduos ocorreu em maio de 2005, quando foram coletadas 818 coleobrocas; o mês de menor ocorrência foi novembro de 2004, quando foram capturadas 96 coleobrocas (Figuras 1, 2 e 3).

\section{pluviométrica \\ Correlação das coleobrocas com a precipitação}

A precipitação pluviométrica pode contribuir diretamente no desenvolvimento e na oferta de alimentos das coleobrocas. De acordo com Whiley \& Schaffer (1997) e Allen et al. (1998), a precipitação pluviométrica pode aumentar a evapotranspiração das mangueiras a qual, como conseqüência, pode causar secamento e queda de ramos ponteiros, contribuindo para um aumento na população de coleobrocas através da deposição de madeira sob as copas. As coleobrocas em geral e os indivíduos da família Curculionidae atingiram o pico populacional durante o mês de maio de 2005, quando foi registrada precipitação pluviométrica de $59 \mathrm{~mm}$; a precipitação variou entre 0,0 a $248 \mathrm{~mm}$ (Figura 4). Bostrichidae e Cerambycidae foram registrados no mês de fevereiro de 2005, quando ocorreu precipitação pluviométrica de $248 \mathrm{~mm}$. Esses resultados diferem dos encontrados por Dall' Oglio \& Peres Filho (1997) que, ao monitorar coleobrocas da família Curculionidae, encontraram o maior pico populacional com precipitação pluviométrica de 19,9 $\mathrm{mm}$.

Não houve correlação entre a precipitação pluviométrica e a flutuação populacional de coleobrocas da família Curculionidae, registrada durante o período de monitoramento, e também não foi constatada significância em nível de $10 \%$ de probabilidade, pelo teste " $t$ "de Student, porém foi positiva para as famílias Bostrichidae e Cerambycidae (Tabela 4). Esses resultados diferem dos encontrados por Müller \& Andreiv (2004) que estudaram coleobrocas em ambientes florestais dos municípios catarinenses de Blumenal e Ilhota e evidenciaram correlação positiva entre as coleobrocas e a precipitação pluviométrica, independentemente da família. Esses autores afirmam ainda que a disponibilidade de hospedeiros no verão é maior, o que explica o aumento do número desses indivíduos nesta época do ano e, por conseqüência, o ciclo biológico desses insetos está associado e regulado por variáveis climáticas.

A família Cerambycidae correlacionou-se positivamente à precipitação pluviométrica. Este fato pode ser explicado pela atividade carpofágica desses indivíduos, pois, de acordo com Garcia \& Corseuil (1999), que estudaram espécies de coleópteros em cultivares de pessegueiros no município de Porto Alegre, Estado do Rio Grande do Sul, a associação de espécies de coleópteros a cultivares de pessegueiros acontece de forma que a ocorrência de um grupo de coleópteros não é influenciada 
pelas cultivares, mas, apesar disso, tem relação direta com a época de maturação dos frutos, e esse fator pode ser influenciado pela precipitação pluviométrica. Dorval \& Peres Filho (2001), estudando vegetação de cerrado, no município de Cuiabá, Estado do Mato Grosso, realizaram correlações simples entre coleópteros e os dados meteorológicos, encontrando resultados significativos para as coleobrocas (Coleoptera: Curculionidae: Scolytinae) Cryptocarenus heveae (Hagedorni) e a precipitação pluviométrica; Cryptocarenus diadematus (Eggers) e as temperaturas mínimas e médias, além da umidade relativa do ar. Já a coleobroca Cryptocarenus seriatus (Eggers) correlacionouse apenas à precipitação pluviométrica.

Dall' Oglio \& Peres Filho (1997), utilizando armadilha etanólica para monitorar coleobrocas da família Curculionidae em plantios de seringueiras, no município de Itiquira, Estado do Mato Grosso, observaram que a maioria das espécies de Scolytinae encontraram condições ambientais favoráveis, independentemente da presença ou ausência de chuva, provavelmente causada pela formação do microclima dessas plantas, fenômeno também muito comum em pomares de manga.

\section{Associação entre as espécies de coleobrocas e as variedades de manga}

Na Tabela 4, pode ser observada a associação entre as espécies de coleobrocas e as variedades de manga cultivadas na área em estudo. As coleobrocas Hypothenemus sp. 1, Hypothenemus sp. 2, Xyleborus sp. 1, Xyleborus sp. 3, Xyleborus sp. 4, Xyleborus sp. 5, Xylosandrus sp., Xylosandrus retusus (Eichhoff), Zygops sp., Achryson surinamum (Linnaeus), Chlorida festiva (L.), Chydarteres dimidiatus dimidiatus (Fabr.), Compsoibidion vanum (Thomson), Hylettus seniculus (Germar), Nesozineus bucki (Breuning), Oxymerus aculeatus aculeatus (Dupont) e Orthostoma chryseis (Bates) foram encontradas associadas às cultivares Tommy Atkins, Keitt, Kent e Palmer.

As espécies Hypocryphalus mangiferae (Stebbing), Marshallius multisignatus (Boheman) e Trachyderes (Trachyderes) succintus succintus (L.) foram encontradas apenas na cultivar Tommy Atkins. Platypus parallelus (Fabr.), Xyleborus sp. 2, Bostrichopsis unicinata (Germar), Eburodacrys assimilis (Gounelle) e Eburodacrys sexmaculata (Olivier) foram associadas especificamente à variedade Palmer. Ataxia obscura (Fabricius), Nesozineus sp., Nyssodrysternum sp. e Paranyssicus conspicillatus (Erichson) foram registrados apenas na variedade Kent, e Hypsioma sp. encontrou-se associada exclusivamente às cultivares Keitt e Kent. Resultados semelhantes foram obtidos por Garcia \& Corseuil (1999), que desenvolveram pesquisa no município de Porto Alegre, Estado do Rio Grande do Sul, para estudar a associação de espécies de coleópteros a cultivares de pessegueiros naquele município. Esses autores afirmaram que a ocorrência de um grupo de coleópteros não é influenciada pelas cultivares, ao contrário de outro grupo de coleópteros, que, pelo seu hábito alimentar, tem relação direta com as épocas de maturação dos frutos.

Os resultados encontrados neste trabalho revelam que a associação de uma determinada espécie de coleobroca a várias cultivares ou a uma cultivar específica pode depender da inter- relação entre a planta e o inseto, e a influência que as variações do ambiente tem sobre ambos. Esse fato pode ter contribuído para um maior destaque das espécies Hylettus seniculus (Germar) (Coleoptera: Cerambycidae) e Hypothenemus sp. 1 e sp. 2. (Coleoptera: Curculionidae: Scolytinae), pois, segundo Silveira Neto et al. (1976) e Paine \& Stephen (1987), a predominância de uma determinada espécie dentro de um povoamento homogêneo e a associação deste inseto a esta espécie vegetal é expressada pela sua adaptabilidade a fatores relacionados à espécie vegetal hospedeira e a características do talhão, como idade, produção de resina, diâmetro, espessura do floema, densidade de plantas e crescimento radial.

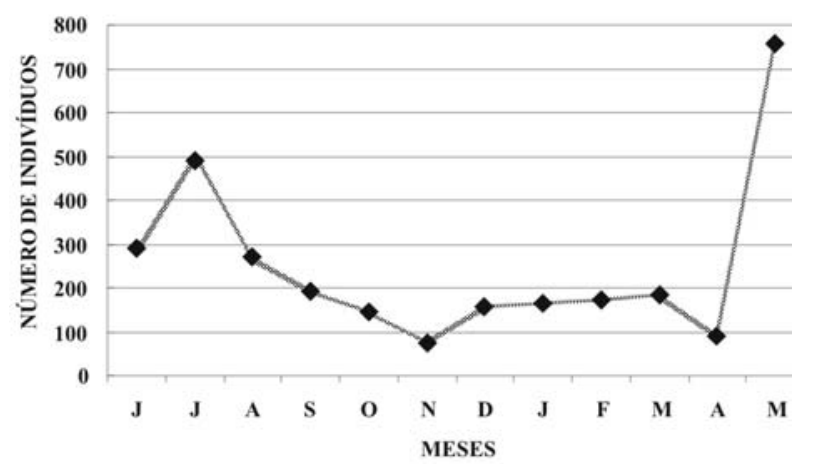

FIGURA 1- Flutuação populacional de Curculionidae coletados com o auxílio da armadilha etanólica Carvalho 47, em área produtora de manga (Mangifera indica), no município de José de Freitas, Estado do Piauí, de junho de 2004 a maio de 2005.

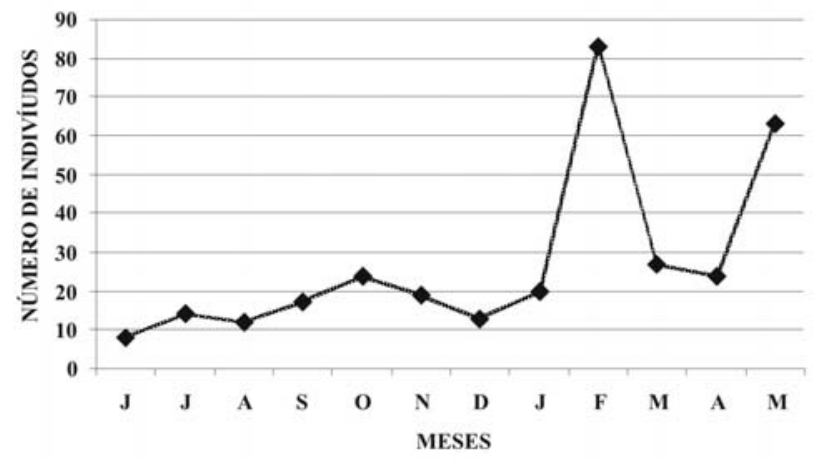

FIGURA 2- Flutuação populacional de Cerambycidae coletados com o auxílio da armadilha etanólica Carvalho 47, em área produtora de manga (Mangifera indica), no município de José de Freitas, Estado do Piauí, de junho de 2004 a maio de 2005. 


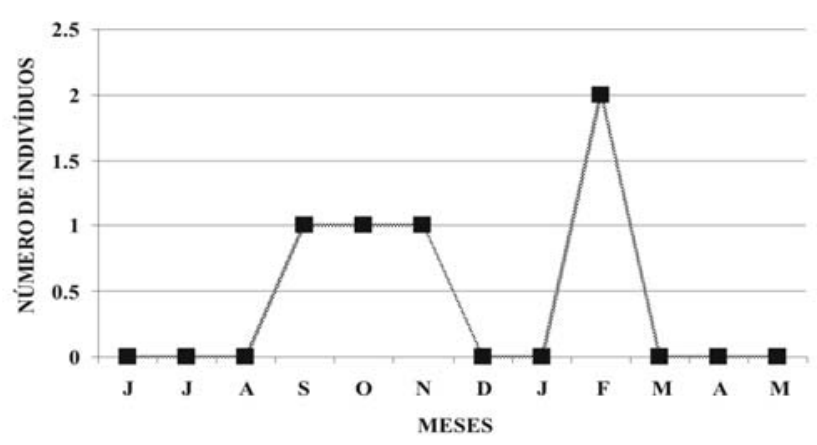

FIGURA 3 - Flutuação populacional de Bostrichidae coletados com o auxílio da armadilha etanólica Carvalho 47, em área produtora de Mangifera indica, no município de José de Freitas, Estado do Piauí, de junho de 2004 a maio de 2005 .

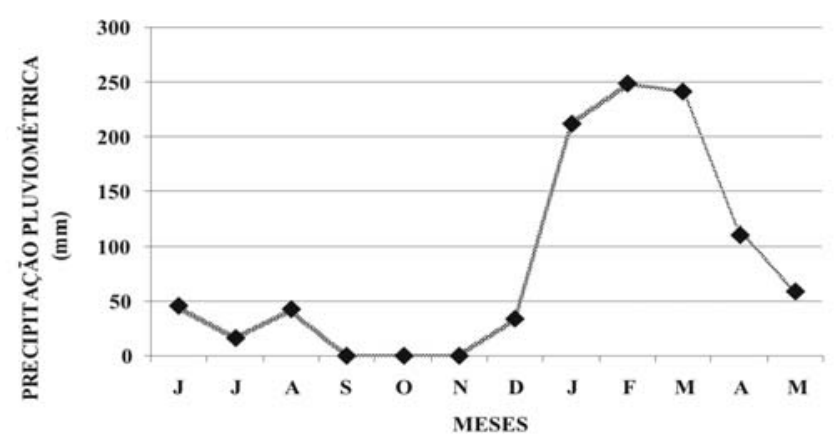

FIGURA 4 - Precipitação Pluviométrica (mm) do município de José de Freitas, Estado do Piauí, de junho de 2004 a maio de 2005.

TABELA 1- Espécies de coleobrocas coletadas com o auxílio da armadilha etanólica Carvalho 47, em área produtora de manga (Mangifera indica), no município de José de Freitas, Estado do Piauí, de junho de 2004 a maio de 2005.

\begin{tabular}{|c|c|c|c|c|c|c|c|c|c|c|c|c|c|}
\hline \multirow[t]{3}{*}{ FAMÍLIAS/ESPÉCIES } & \multicolumn{7}{|c|}{2004} & \multicolumn{5}{|c|}{2005} & \multirow[t]{2}{*}{ Total } \\
\hline & \multicolumn{12}{|c|}{ Meses } & \\
\hline & $\mathbf{J}$ & $\mathbf{J}$ & $\mathbf{A}$ & $\mathbf{S}$ & $\mathbf{O}$ & $\mathbf{N}$ & D & $\mathbf{J}$ & $\mathbf{F}$ & $\mathbf{M}$ & $\mathbf{A}$ & $\mathbf{M}$ & \multirow[b]{3}{*}{5} \\
\hline \multicolumn{13}{|l|}{ BOSTRICHIDAE } & \\
\hline Bostrichopsis unicinata (Germar) & 0 & 0 & 0 & 1 & 1 & 1 & 0 & 0 & 2 & 0 & 0 & 0 & \\
\hline Subtotal & $\mathbf{0}$ & $\mathbf{0}$ & $\mathbf{0}$ & 1 & 1 & 1 & $\mathbf{0}$ & $\mathbf{0}$ & 2 & $\mathbf{0}$ & $\mathbf{0}$ & $\mathbf{0}$ & 5 \\
\hline \multicolumn{14}{|l|}{ CERAMBYCIDAE } \\
\hline$\overline{\text { Achryson surinamum (L.) }}$ & 0 & 3 & 2 & 1 & 2 & 4 & 2 & 1 & 2 & 0 & 1 & 0 & 18 \\
\hline Ataxia obscura (Fabr.) & 0 & 0 & 0 & 0 & 0 & 0 & 0 & 1 & 1 & 0 & 0 & 0 & 2 \\
\hline Chlorida festiva (Linnaeus) & 0 & 1 & 3 & 2 & 5 & 0 & 1 & 1 & 1 & 0 & 0 & 0 & 14 \\
\hline Chydarteres dimidiatus dimidiatus (Fabr.) & 0 & 0 & 1 & 0 & 0 & 0 & 0 & 1 & 6 & 1 & 0 & 0 & 9 \\
\hline Compsoibidion vanum (Thomson) & 4 & 4 & 3 & 4 & 10 & 5 & 7 & 4 & 2 & 1 & 0 & 0 & 44 \\
\hline Eburodacrys assimilis (Gounelle) & 0 & 0 & 0 & 0 & 0 & 0 & 1 & 0 & 0 & 0 & 0 & 0 & 1 \\
\hline Eburodacrys sexmaculata (Olivier) & 0 & 0 & 0 & 1 & 0 & 0 & 0 & 1 & 0 & 0 & 1 & 4 & 7 \\
\hline Hylettus seniculus (Germar) & 0 & 1 & 0 & 4 & 3 & 8 & 2 & 8 & 49 & 22 & 20 & 36 & 153 \\
\hline Hypsioma sp. & 0 & 0 & 1 & 1 & 1 & 0 & 0 & 0 & 0 & 1 & 0 & 0 & 4 \\
\hline Leptostylus sp. & 0 & 0 & 0 & 0 & 0 & 0 & 0 & 0 & 0 & 1 & 0 & 6 & 7 \\
\hline Nesozineus bucki (Breuning) & 0 & 0 & 0 & 0 & 0 & 1 & 0 & 0 & 17 & 0 & 0 & 0 & 18 \\
\hline Nesozineus sp. & 0 & 1 & 0 & 0 & 1 & 0 & 0 & 1 & 0 & 0 & 0 & 0 & 3 \\
\hline Nyssodrysternum sp. & 0 & 0 & 0 & 0 & 1 & 1 & 0 & 1 & 0 & 0 & 0 & 4 & 7 \\
\hline Orthostoma chryseis & 4 & 3 & 1 & 3 & 1 & 0 & 0 & 0 & 1 & 1 & 1 & 5 & 20 \\
\hline Oxymerus aculeatus aculeatus (Dupont) & 0 & 0 & 0 & 0 & 0 & 0 & 0 & 1 & 3 & 0 & 0 & 4 & 8 \\
\hline Paranyssicus conspicillatus (Erichson) & 0 & 0 & 1 & 1 & 0 & 0 & 0 & 0 & 1 & 0 & 0 & 4 & 7 \\
\hline Trachyderes (Trachyderes) succintus succintus (L.) & 0 & 1 & 0 & 0 & 0 & 0 & 0 & 0 & 0 & 0 & 1 & 0 & 2 \\
\hline Subtotal & 8 & 14 & 12 & 17 & 24 & 19 & 13 & 20 & 83 & 27 & 24 & 63 & 324 \\
\hline \multicolumn{14}{|l|}{ CURCULIONIDAE } \\
\hline Hypocryphalus mangiferae (Stebbing) & 0 & 0 & 0 & 0 & 0 & 2 & 0 & 0 & 0 & 0 & 0 & 0 & 2 \\
\hline Hypothenemus sp. 1 & 52 & 41 & 87 & 58 & 50 & 21 & 14 & 37 & 32 & 36 & 13 & 60 & 501 \\
\hline Hypothenemus sp. 2 & 196 & 392 & 55 & 43 & 26 & 16 & 70 & 80 & 80 & 76 & 31 & 512 & 1.577 \\
\hline Marshallius multisignatus (Boheman) & 0 & 0 & 0 & 0 & 0 & 0 & 0 & 0 & 0 & 1 & 0 & 0 & 1 \\
\hline Platypus parallelus (Fabr.) & 0 & 0 & 0 & 0 & 0 & 0 & 3 & 0 & 0 & 0 & 0 & 0 & 3 \\
\hline Xyleborus sp. 1 & 0 & 1 & 6 & 11 & 6 & 2 & 1 & 5 & 0 & 1 & 0 & 0 & 33 \\
\hline Xyleborus sp. 2 & 0 & 0 & 0 & 0 & 0 & 1 & 0 & 0 & 0 & 0 & 0 & 0 & 1 \\
\hline Xyleborus sp. 3 & 0 & 6 & 0 & 6 & 10 & 0 & 1 & 15 & 22 & 34 & 22 & 44 & 160 \\
\hline Xyleborus sp. 4 & 20 & 25 & 56 & 37 & 11 & 8 & 5 & 7 & 19 & 29 & 18 & 76 & 311 \\
\hline Xyleborus sp. 5 & 0 & 3 & 0 & 3 & 0 & 0 & 2 & 0 & 3 & 0 & 0 & 28 & 39 \\
\hline Xylosandrus retusus (Eichhoff) & 0 & 3 & 11 & 22 & 39 & 22 & 37 & 6 & 6 & 2 & 0 & 4 & 152 \\
\hline Xylosandrus sp. & 20 & 16 & 45 & 7 & 1 & 2 & 22 & 10 & 4 & 2 & 5 & 23 & 157 \\
\hline Zygops sp. & 4 & 2 & 10 & 4 & 4 & 2 & 2 & 6 & 6 & 2 & 1 & 8 & 51 \\
\hline Subtotal & 292 & 489 & 270 & 191 & 147 & 76 & 157 & 166 & 172 & 183 & 90 & 755 & 2.988 \\
\hline Total & 300 & 503 & 282 & 209 & 172 & 96 & 170 & 186 & 257 & 210 & 114 & 818 & 3.317 \\
\hline
\end{tabular}

1, 2, 3, 4 e 5 - Espécies não-identificadas, porém distintas.

Rev. Bras. Frutic., Jaboticabal - SP, v. 30, n. 2, p. 348-355, Junho 2008 
TABELA 2 - Freqüências por família (F. F.) e por espécie (F. E.) de coleobrocas coletadas com o auxílio da armadilha etanólica CARVALHO 47, em área produtora de manga (Mangifera indica), no município de José de Freitas, Estado do Piauí, de junho de 2004 a maio de 2005.

\begin{tabular}{|c|c|c|}
\hline FAMIILIAS/ ESPÉCIES & F. F.(\%) & F. E. (\%) \\
\hline \multicolumn{3}{|l|}{ BOSTRICHIDAE } \\
\hline Bostrichopsis unicinata (Germar) & 100 & 0,15 \\
\hline Subtotal & 100 & 0,1 \\
\hline \multicolumn{3}{|l|}{ CERAMBYCIDAE } \\
\hline 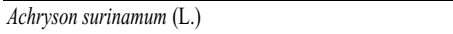 & 5,56 & 0,54 \\
\hline Ataxia obscura (Fabr.) & 0,62 & 0,06 \\
\hline Chlorida festiva (L.) & 4,32 & 0,42 \\
\hline Chydarteres dimidiatus dimidiatus (Fabr.) & 2,78 & 0,27 \\
\hline Compsoibidion vanum (Thomson) & 13,58 & 1,33 \\
\hline Eburodacrys assimilis (Gounelle) & 0,31 & 0,03 \\
\hline Eburodacrys sexmaculata (Olivier) & 2,16 & 0,21 \\
\hline Hylettus seniculus (Germar) & 47,22 & 4,61 \\
\hline Hypsioma sp. & 1,23 & 0,12 \\
\hline Leptostylus sp. & 2,16 & 0,21 \\
\hline Nesozineus bucki (Breuning) & 5,56 & 0,54 \\
\hline Nesozineus sp. & 0,93 & 0,09 \\
\hline Nyssodrysternum sp. & 2,16 & 0,21 \\
\hline Orthostoma chryseis (Bates) & 6,17 & 0,60 \\
\hline Oxymerus aculeatus aculeatus (Dupont) & 2,47 & 0,24 \\
\hline Paranyssicus conspicillatus (Erichson) & 2,16 & 0,21 \\
\hline Trachyderes (Trachyderes) s uccintus succintus (L.) & 0,62 & 0,06 \\
\hline $\begin{array}{ll}\text { Subtotal } \\
\end{array}$ & 100,00 & 9,8 \\
\hline \multicolumn{3}{|l|}{ CURCULIONIDAE } \\
\hline Hypocryphalus mangiferae (Stebbing) & 0,07 & 0,06 \\
\hline Hypothenemus sp. 1 & 16,77 & 15,10 \\
\hline Hypothenemus sp. 2 & 52,78 & 47,54 \\
\hline Marshallius multisignatus (Boheman) & 0,03 & 0,03 \\
\hline Platypus parallelus (Fabr.) & 0,10 & 0,09 \\
\hline Xyleborus sp. 1 & 1,10 & 0,99 \\
\hline Xyleborus sp. 2 & 0,03 & 0,03 \\
\hline Xyleborus sp. 3 & 5,35 & 4,82 \\
\hline Xyleborus sp. 4 & 10,41 & 9,38 \\
\hline Xyleborus sp. 5 & 1,31 & 1,18 \\
\hline Xylosandrus retusus (Eichhoff) & 5,09 & 4,58 \\
\hline Xylosandrus sp. & 5,25 & 4,73 \\
\hline Zygops sp. & 1,71 & 1,54 \\
\hline Subtotal & 100,00 & 90,1 \\
\hline Total & - & 100,00 \\
\hline
\end{tabular}

1, 2, 3, 4 e 5 - Espécies não-identificadas, porém distintas.

TABELA 3 - Coeficientes de correlações (r) e significância (t) entre as famílias de coleobrocas coletadas em área produtora de manga (Mangifera indica), no município de José de Freitas, Estado do Piauí, de junho de 2004 a maio de 2005.

\begin{tabular}{lcc}
\hline CORRELAÇÕES & $\mathrm{r}$ & $\mathrm{t}$ \\
\hline PP $(\mathrm{mm}) /$ Bostrichidae & 0,11 & $0,34^{\mathrm{ns}}$ \\
PP $(\mathrm{mm}) /$ Cerambycidae & 0,50 & 1,85 \\
PP $(\mathrm{mm}) /$ Curculionidae & $-0,18$ & $-0,58^{\mathrm{ns}}$
\end{tabular}

PP (mm)-Precipitação, em milímetros; r-Coeficiente de Correlação Simples; t-teste " $t$ "de Student; ns-Teste " $t$ " não- significativo (para 10 graus de liberdade, " $\mathrm{t}$ " tabelado a $10 \%=1,81$ ).
TABELA 4 - Coleobrocas associadas a variedades de manga (Mangifera indica), no município de José de Freitas, Estado do Piauí.

\begin{tabular}{|c|c|}
\hline FAMÍLIAS/ESPÉCIES & VARIEDADES \\
\hline \multicolumn{2}{|l|}{ BOSTRICHIDAE } \\
\hline Bostrichopsis unicinata (Germar) & Palmer \\
\hline \multicolumn{2}{|l|}{ CERAMBYCIDAE } \\
\hline Achryson surinamum (L.) & Tommy Atkins; Keitt; Kent e Palmer \\
\hline Ataxia obscura (Fabr.) & Kent \\
\hline Chlorida festiva (L.) & Tommy Atkins; Keitt; Kent e Palmes \\
\hline Chydarteres dimidiatus dimidiatus (Fabr.) & Tommy Atkins; Keitt; Kent e Palmer \\
\hline Compsoibidion vanum (Thomson) & Tommy Atkins; Keitt; Kent e Palmer \\
\hline Eburodacrys assimilis (Gounelle) & Palmer \\
\hline Eburodacrys sexmaculata (Olivier) & Palmer \\
\hline Hylettus seniculus (Germar) & Keitt e Kent \\
\hline Hypsioma sp. & Tommy Atkins; Keitt; Kent e Palmes \\
\hline Leptostylus sp. & Palmer \\
\hline Nesozineus bucki (Breuning) & Kent \\
\hline Nesozineus sp. & Tommy Atkins; Keitt; Kent e Palmer \\
\hline Nyssodrysternum sp. & Kent \\
\hline Orthostoma chryseis (Bates) & Tommy Atkins; Keitt; Kent e Palmeı \\
\hline Oxymerus aculeatus aculeatus (Dupont) & Tommy Atkins; Keitt; Kent e Palmer \\
\hline Paranyssicus conspicillatus (Erichson) & Kent \\
\hline Trachyderes (Trachyderes) succintus succintus (L.) & Tommy Atkins \\
\hline \multicolumn{2}{|l|}{ CURCULIONIDAE } \\
\hline Hypocryphalus mangiferae (Stebbing) & Tommy Atkins \\
\hline Hypothenemus sp. 1 & Tommy Atkins; Keitt; Kent e Palmes \\
\hline Hypothenemus sp. 2 & Tommy Atkins; Keitt; Kent e Palmes \\
\hline Marshallius multisignatus (Boheman) & Tommy Atkins \\
\hline Platypus parallelus (Fabr.) & Palmer \\
\hline Xyleborus sp. 1 & Tommy Atkins; Keitt; Kent e Palmer \\
\hline Xyleborus sp. 2 & Palmer \\
\hline Xyleborus sp. 3 & Tommy Atkins; Keitt; Kent e Palmes \\
\hline Xyleborus sp. 4 & Tommy Atkins; Keitt; Kent e Palmes \\
\hline Xyleborus sp. 5 & Tommy Atkins; Keitt; Kent e Palmes \\
\hline Xylosandrus retusus (Eichhoff) & Tommy Atkins; Keitt; Kent e Palmer \\
\hline Xylosandrus sp. & Tommy Atkins; Keitt; Kent e Palmer \\
\hline Zygops sp. & Tommy Atkins; Keitt; Kent e Palmer \\
\hline
\end{tabular}

1, 2, 3, 4 e 5 - Espécies não-identificadas, porém distintas.

\section{CONCLUSÕES}

1-Coleobrocas das famílias Bostrichidae, Cerambycidae e Curculionidae estão associadas às cultivares de manga cultivadas no município de José de Freitas, Estado do Piauí.

2-Hylettus seniculus (Germar) destacou-se entre os Cerambycidae com o maior número de indivíduos coletados, Hypothenemus sp. 1, Hypothenemus sp. 2 e Xyleborus sp 4 foram os curculionidae mais abundantes nesse período de estudo.

3-O fator precipitação pluviométrica está correlacionado positivamente à coleta de indivíduos da família Cerambycidae.

\section{AGRADECIMENTOS}

À Fazenda Frutan Brasil e seus funcionários, em especial ao Engenheiro Agrônomo Lívio de Sousa Moura, pelo apoio fornecido durante os trabalhos de campo. Ao Dr. Sérgio Ide, do 
Instituto Biológico de São Paulo, pela classificação dos Bostrichidae e Curculionidae, e ao Dr. Ubirajara R. Martins, do Museu de Zoologia da USP, pela determinação das espécies de Cerambycidae nesta pesquisa.

\section{REFERÊNCIAS}

ABREU, R.L. S.; SALES-CAMPOS, C.; HANADA, R.E.; VASCONCELOS, F.J.; FREITAS, J.A. Avaliação de danos por insetos em toras estocadas em indústrias madeireiras de Manaus, Amazonas, Brasil. Árvore, Viçosa, v.26, n.6, p. 789-796, 2002.

ALLEN, R.G.; PEREIRA, L.S.; RAES, D.; SMITH, M. Crop evapotranspiration, guideliness for computing crop water requeriments. Rome: $\mathrm{FAO}, 1998.300 \mathrm{p}$.

BATRA, L.R. Ecology of ambrosia fungi and their dissemination by beetles. Transactions of the Kansas Academy of Science, Lawrence, v. 66, n. 2, p. 213-36, 1963.

CARVALHO, A.O.R. Análise faunística de coleópteros coletados em plantas de Eucalyptus urophylla S.T. Blake e Eucalyptus saligna Sm. 1984. 105 f. Dissertação (Mestrado em Entomologia) - Universidade de São Paulo, Escola Superior de Agricultura "Luiz de Queiroz", Piracicaba, 1984.

CARVALHO, A.G.; ROCHA, M.P.; SILVA, C.A.M.; LUNZ, A.M. Variação sazonal de Scolytidae (Coleoptera) numa comunidade de floresta natural de Seropédica, RJ. Floresta e Ambiente, Seropédica, v.3, n. 2, p.9- 14, 1996.

CARVALHO, A.G.; ROCHA, M. P.; SILVA, C.A.M.; LUNZ, A.M. Análise da flutuação populacional de Cerambycidae em uma mata nativa na região de Seropédica, RJ. In: CONGRESSO BRASILEIRODEENTOMOLOGIA,16., ENCONTRONACIONAL DE FITOSSANITARISTAS, 7., 1997. Salvador. Resumos... Salvador: SEB; EMBRAPACNPMF, 1997. p. 247.

DALL'OGLIO, O. T.; PERES FILHO. O. Levantamento e flutuação de populacional de coleobrocas em plantios homogêneos de seringueira em Itiquira-MT. Scientia Forestalis, Piracicaba, v.51, n. 2, p.49-58, 1997.

FERRAZ, F. C.; CARVALHO, A. G.; COUTINHO, C. L.; SOUZA, N. J. Eficiência de armadilhas etanólicas para levantamentos de coleópteros do reflorestamento de Eucalyptus citriodora em Pinheiral, RJ. Floresta e Ambiente, Seropédica, v. 6, n.1, p. 159$162,1999$.

FERRAZ, F.C.; CARVALHO,A. G.; MAURICIO, E. G. Coleópteros degradadores de madeira, coletados com armadilhas de impacto em pomar de citros no município de Pinheiral, Rio de Janeiro. UFRRJ. Floresta e Ambiente, Seropédica, v.7, n. 1, p. 88-92, 2000.
FLECHTMANN, C.A. H.; GASPARETO, C.L. Scolytidae em pátio de serraria da fábrica Paula Souza (Botucatu/SP) e fazenda Rio Claro (Lençóis Paulista/SP). Scientia Forestalis, Piracicaba, v.51, n. 2, p.61-75, 1997.

FLECHTMANN, C.A.H.; OTTATI, A.L.T.; BERISFORD, C.W. Attraction of ambrosia beetles (Coleoptera: Scolytidae) to different tropical pine species in Brazil. Environmental Entomology, Lanhan, v. 28, n. 2, p. 649-658, 1999.

FLECHTMANN, C.A.H.; OTTATI, A.L.T.; BERISFORD, C.W. Ambrosia and bark beetles (Scolytidae: Coleoptera) in pine and eucaypt stands in southern Brazil. Forest Ecology and Management, Amsterdan, v. 142, n. 1, p. 183-191, 2001.

GALLO,D.; NAKANO, O.; SILVEIRANETO, S.; CARVALHO, R. P. L.; BAPTISTA, G. C.; BERTI FILHO, E.; PARRA, J. R. P.; ZUCCHI, R. A.; ALVES, S. B.; VENDRAMIM, J. D.; MARCHINI, L. C.; LOPES, J. R. S.; OMOTO, C. Entomologia agrícola. Piracicaba: FEALQ. 2002. 920p. . (Biblioteca de Ciências Agrárias Luiz de Queiroz, 10)

GARCIA, F. R.M.; CORSEUIL, E. Flutuação populacional de cerambicídeos e escarabeídeos (COLEOPTERA) em pomares de pessegueiros no município de Porto Alegre, Rio Grande do Sul. Revista da Faculdade de Zootecnia, Veterinária e Agronomia, Uruguaiana, v. 5/6, n. 1, p. 61-70, 1999.

GRAY, B. Economic tropical forest entomology. Annual Review Entomology, Palo Alto, v. 17, p. 313-354, 1972.

HOSKING, G. P. Xyleborus saxeseni, its life-history and flight behaviour in New Zealand. New Zealand Journal of Forestry Science, Rotoura, v. 3, n.1, p. 37-53, 1977.

LIMA, V. C. A. Contribuição ao estudo de Marshallius anacardii sp. praga do cajueiro em Pernambuco (Coleoptera, Curculionidae). In: REUNIÃO DE PESQUISA DA UNIVERSIDADE FEDERAL RURAL DE PERNAMBUCO, 4. , 1979. Recife. Anais... p.115-148.

LIMA, M. G. Estimativa da temperatura do ar no Piauí. Teresina: UFPI, 2002. 48p.

MORALES, N. E.; ZANUNCIO, J. C.; MARQUES, E. N.; PERTISSOLI, D.; COUTO, L. Índices populacionais de besouros Scolytidae em reflorestamento de Eucalyptus grandis W. Hill ex Maiden no município de Antônio Dias, Minas Gerais. Árvore, Viçosa, v. 23, n. 2, p. 359-363, 1996.

NAKANO, O.; LEITE, C.A. Armadilhas para insetos: pragas agrícolas e domésticas. Piracicaba: FEALQ, 2000. 76p. (Biblioteca de Ciências Agrárias Luiz de Queiroz, 7). 
PAINE, T.D.; F.M. STEPHEN. The relationship of tree height and crown class to the induced plant defenses of loblolly pine. Canadian Journal of Botany, Guelph, v. 65, n. 2, 2090-2092, 1987.

SILVEIRA NETO, S.; NAKANO, O.; BARBIN, D. Manual de ecologia dos insetos. São Paulo: Agronômica Ceres, 1976, 419p.

VITÉ, J.P. Silviculture and the management of bark beetle pests. In: CONFERENCE ONECOLOGICALANIMAL CONTROL BY HABITAT MANAGEMENT, 13., 1971. p.155-68.
WHILEY, A.W.; SCHAFFER, B. Stress physiology. In: LITZ, R.E. (Ed.). The mango: botany, production and uses. Florida: $\mathrm{CAB}$ International, 1997. cap. 5, p.147-173.

ZANUNCIO, J.C.; M.F.SOSSAI; C.A.H. FLECHTMANN; T.V. ZANUNCIO; E.M. GUIMARÃES \& M.C. ESPINDULA. Plants of an Eucalyptus clone damage by Scolytidae and Platypodidae (Coleoptera). Pesquisa Agropecuária Brasileira, Brasília, v.40, n.2, p. 513-515, 2005. 